\title{
Application virtual machines for support of educational process at the department of the university
}

\section{Braginsky Aleksandr Isaakovich}

Russian Federation, Ph. D., Associate Professor, Department of «Automated Control Systems».

Moscow Automobile \& Road construction State Technical University, 125319, Russian Federation, Moscow, Leningradsky prospekt, 64. Tel.: +7 (499) 151-64-12. http://www.madi.ru

abra@mail.ru

\section{Gapshin Nikolay Vladimirovich}

Russian Federation, Senior Lecturer, Department of «Automated Control Systems».

Moscow Automobile \& Road construction State Technical University, 125319, Russian Federation, Moscow, Leningradsky prospekt, 64. Tel.: +7 (499) 151-64-12. http://www.madi.ru

\section{Golubkova Valentina Borisovna}

Russian Federation, Ph. D., Associate Professor, Department of «Automated Control Systems».

Moscow Automobile \& Road construction State Technical University, 125319, Russian Federation, Moscow, Leningradsky prospekt, 64. Tel.: +7 (499) 151-64-12. http://www.madi.ru

vb.citrus@mail.ru

\section{Vinogradov Vadim Alekseevich}

Russian Federation, Senior Lecturer, Department of «Automated Control Systems».

Moscow Automobile \& Road construction State Technical University, 125319, Russian Federation, Moscow, Leningradsky prospekt, 64. Tel.: +7 (499) 151-64-12. http://www.madi.ru

\section{vvgarage@mail.ru}

\begin{abstract}
The article deals with issues connected with technical support of educative process at the department of the university specialized in IT. Arguments for and brief analysis of technical support requirements are being provided. A solution is being proposed that is based upon usage of virtualization technology and know-how, developed by VMware Company. The most typical objectives of education process support and their practical implementation are being shown.
\end{abstract}

Keywords: virtualization, education standards, virtual server, VMware. 
ISSN 2306-1561

Автоматизация и управление в технических системах (АУТС) 2014. - №1.1(8). - C. 14-24.

DOI: $10.12731 / 2306-1561-2014-1-2$

\section{УДК 004.8}

\section{Применение виртуальных машин для технического обеспечения учебного процесса на кафедре ВУЗа}

\section{Брагинский Александр Исаакович}

Российская Федерация, кандидат технических наук, доцент кафедры «Автоматизированные системы управления».

ФГБОУ ВПО «Московский автомобильно-дорожный государственный технический университет (МАДИ)», 125319, Российская Федерация, г. Москва, Ленинградский проспект, д.64, Тел.: +7 (499) 151-64-12, http://www.madi.ru

a_bra@mail.ru

\section{Гапшин Николай Владимирович}

Российская Федерация, старший преподаватель кафедры «Автоматизированные системы управления».

ФГБОУ ВПО «Московский автомобильно-дорожный государственный технический университет (МАДИ)», 125319, Российская Федерация, г. Москва, Ленинградский проспект, д.64, Тел.: +7 (499) 151-64-12, http://www.madi.ru

\section{Голубкова Валентина Борисовна}

Российская Федерация, кандидат технических наук, доцент кафедры «Автоматизированные системы управления».

ФГБОУ ВПО «Московский автомобильно-дорожный государственный технический университет (МАДИ)», 125319, Российская Федерация, г. Москва, Ленинградский проспект, д.64, Тел.: +7 (499) 151-64-12, http://www.madi.ru

\section{vb.citrus@mail.ru}

\section{Виноградов Вадим Алексеевич}

Российская Федерация, старший преподаватель кафедры «Автоматизированные системы управления».

ФГБОУ ВПО «Московский автомобильно-дорожный государственный технический университет (МАДИ)», 125319, Российская Федерация, г. Москва, Ленинградский проспект, д.64, Тел.: +7 (499) 151-64-12, http://www.madi.ru 
Аннотация. В статье рассмотрены вопросы, технического обеспечения учебного процесса на кафедре ВУЗа, связанной с обучением в области ИТ-технологий. Приводятся обоснование и краткий анализ сформулированных требований к техническому обеспечению. Предлагается решение, основанное на использовании технологии виртуализации и разработок компании VMware. Рассматриваются наиболее типичные задачи обеспечения учебного процесса и их практическая реализация.

Ключевые слова: технология виртуализации, стандарты образования, виртуальный сервер, VMware.

\section{1. Введение}

В ходе учебного процесса на кафедре ВУЗа, связанной с преподаванием ИТтехнологий, необходимо обеспечивать (на аппаратном и программном уровнях) решение следующих вопросов $[1,2]$ :

- Приобретение знаний и умений, связанных с установкой, настройкой, функционированием и эксплуатацией различных операционных систем (OC) с учетом сетевого взаимодействия (как в локальных, так и в глобальных вычислительных сетях).

- Приобретение знаний и умений, связанных с установкой, настройкой, функционированием и эксплуатацией различного программного обеспечения (ПО) с учетом сетевого взаимодействия (как в локальных, так и в глобальных вычислительных сетях).

- Приобретение знаний и умений, связанных с написанием, отладкой и эксплуатацией ПО в различных средах, с использованием различных языков программирования. Тут, как и в предыдущих пунктах невозможно обойтись без присутствия различного сетевого взаимодействия.

Безусловно приведенный перечень достаточно условен и может быть расширен или сформулирован по-другому. В данном случае, важно следующее - во всех пунктах присутствуют требования к наличию разнородного ПО, действующего в локальных и глобальных вычислительных сетях. На эти требования, в свою очередь, накладываются требования, связанные со спецификой учебного процесса:

Наличие большого количество различных учебных дисциплин, имеющих в качестве обеспечения различное ПО, часто конфликтующее между собой.

Необходимость обновления ПО с разной периодичностью для различных учебных дисциплин.

Учет внешних факторов, заключающихся в необходимости оперативного реагирования на изменяющиеся требования к учебному процессу, введения новых дисциплин, появления нового ПО и т. д.

Приведенные требования, конечно, не исчерпывают все проблемы технического и программного обеспечения учебного процесса изучения ИТ-технологий. Их подробное рассмотрение выходит за рамки настоящей статьи. Отчасти эти вопросы были рассмотрены в [5]. 
Существенным приходится признать, и то, что постоянное развитие информационных технологий вызывает быстрое моральное старение существующего компьютерного оборудования. Ежегодно ВУЗ вынужден обновлять до $30 \%$ компьютерного парка. В условиях постоянного дефицита бюджета эти расходы не посильны и стимулируют поиск альтернативных вариантов.

На наш взгляд, использование технологии виртуальных машин позволяет удовлетворить практически все вышеприведенные требования. Остановимся на этом поподробнее.

\section{2. Технология виртуализации}

Виртуализация компьютеров и серверов - это программное решение, которое позволяет использовать ресурсы одного физического компьютера, чтобы получить несколько разделённых между собой окружений, каждое из которых представляет собой виртуализированный компьютер (сервер) [5, 6, 7].

Существует несколько программных платформ для виртуализации. Это продукты компаний VMware [3], Microsoft, Citrix, Xen, Parallels. Программное обеспечение этих производителей предназначено для использования, как на различных аппаратных серверных платформах, так и на базе обычных персональных компьютерах. Одной из первых разработкой программного обеспечения для виртуализации начала компания VMware, на сегодняшний день, являющаяся признанным лидером в этой области. Технологический уровень разработок VMware, де-факто, признан промышленным стандартом аппаратно-программной виртуализации.

Программные продукты VMware, a их количество приближается к сотне, позволяют виртуализировать сервера и рабочие станции с любой операционной системой, и на практически любой современной аппаратной платформе. Производители компьютерного оборудования не остались в стороне, и сегодня предлагают достаточно большой выбор «железа» оптимизированного для использования средств виртуализации.

Флагманскими продуктами VMware являются VMware ESX/ESXi - гипервизоры, устанавливающиеся на "голое" железо (bare metal). На текущий момент последней версией продукта является версия 5.5, выпущенная в начале 2013 года. Гипервизор является основой для виртуализации серверов, он позволяет разделять ресурсы таким образом, чтобы создавать отдельные, независимые среды для множества операционных систем на одном физическом сервере. Однако сам по себе гипервизор имеет весьма ограниченный круг возможностей, для реализации же всех преимуществ требуются решения, которые включает как средства виртуализации, так и средства управления инфраструктурой (vCenter). Это комплексное решение называется vSphere.

Анализ эффективности использования серверного оборудования показывает, что большую часть рабочего времени загрузка составляет около 5-8\% от максимальной, в нерабочее же время серверы просто простаивают, нагревая воздух. При использовании VMware [3] vSphere [4] возможно оперативно перенести на один сервер нагрузку с нескольких серверов, включая не только приложения, но и операционные системы. 
Производительность современных серверов делает крайне неэффективной популярную ранее концепцию "одна задача - один сервер". Благодаря виртуализации теперь можно использовать новую концепцию "одна задача - одна виртуальная машина" (рисунок 1).

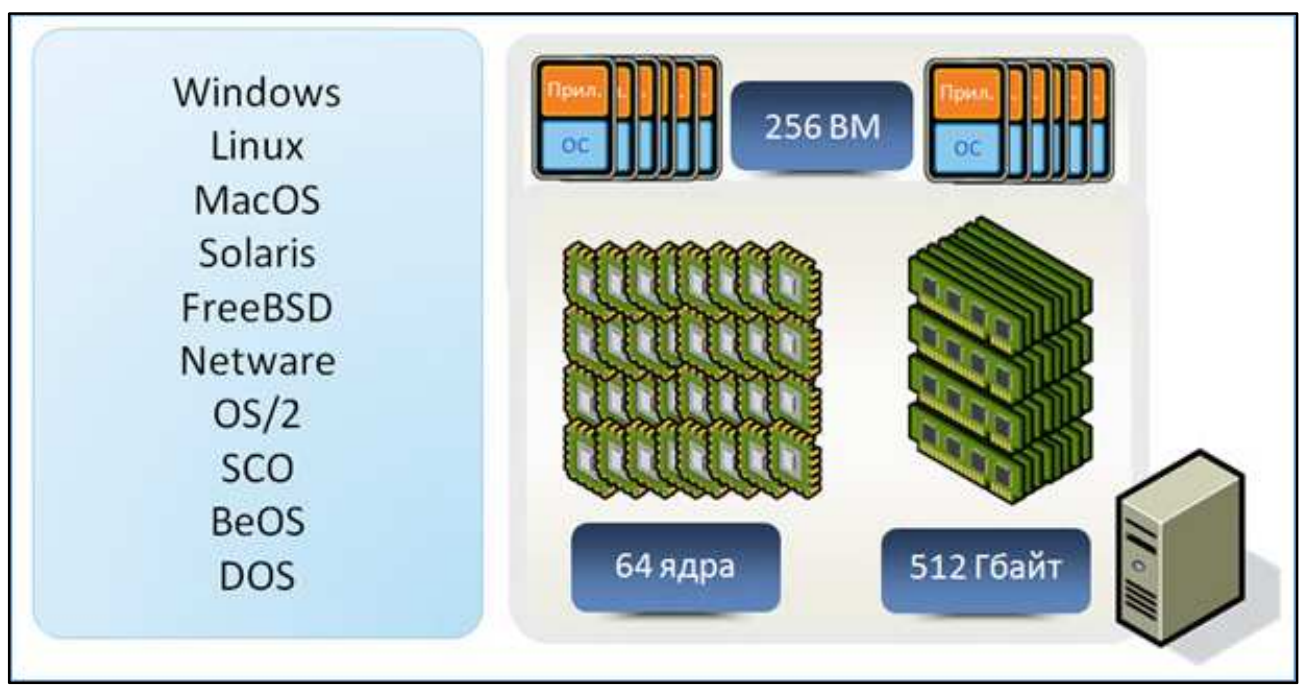

\section{Рисунок 1 - Концепция виртуализации «одна задача - одна виртуальная машина»}

Таким образом, решается проблема совместимости различного ПО - далеко не все приложения можно запустить в одном экземпляре операционной системы. Кроме того, часто в инфраструктуре используются старые приложения, которые уже не совместимы с текущими версиями ОС, а установка старых версий не поддерживается на новом оборудовании.

Виртуализация решает и эту задачу - в виртуальной машине ESX можно запустить даже Windows NT 4.0 или MS-DOS. Конечно, технология виртуализации требует дополнительных затрат ресурсов серверного оборудования, но на текущий момент они составляют 1-3\% от доступной мощности. Для централизованного управления и мониторинга служит специальный продукт - VMware vCenter (рисунок 2).

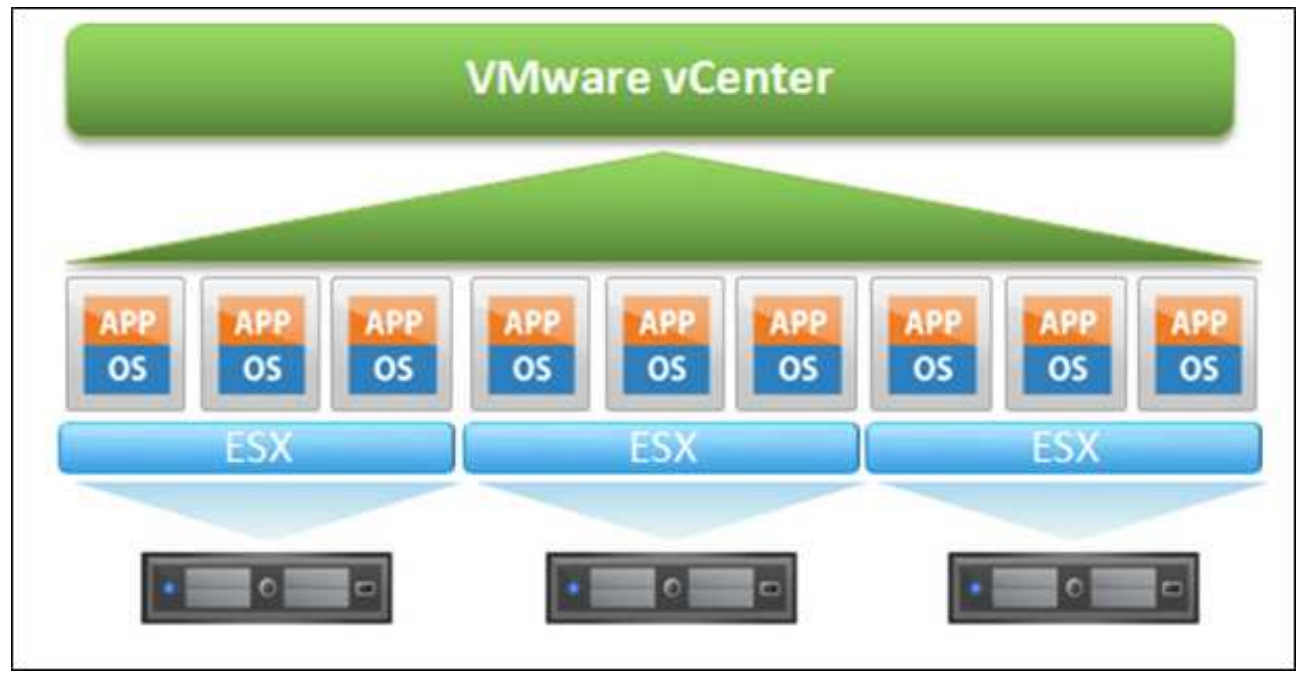

Рисунок 2 - Управление виртуальными серверами из единого центра 
Помимо мониторинга и создания виртуальных серверов, vCenter обеспечивает реализацию таких возможностей как перемещение виртуальных машин между физическими серверами, миграцию дисковых ресурсов, создание мгновенных снимков, развертывание виртуальных машин из шаблонов и прочих дополнительных функций VMware vSphere.

Оба варианта гипервизоров (ESX и ESXi) имеют одинаковый, с точки зрения, виртуальных машин функционал, однако реализации их различна. В состав ESX входит сервисная консоль для управления гипервизором, а ESXi такой консоли не имеет (за счет этого размер его гораздо меньше, а управление возможно только через vCenter, vSphere Client или скрипты на управляющей машине). Если ESX выглядит для пользователя как операционная система, то ESXi скорее напоминает BIOS материнской платы. Установка и первичная настройка ESXi очень проста, а использование Embedded версии (поставляемой вместе с сервером) позволяет развернуть систему виртуализации за считанные минуты. Для пользователей доступна также бесплатная версия ESXi, которая имеет ряд ограничений - например, нет поддержки централизованного управления, а также "enterprise" возможностей vSphere - vMotion, HA, DRS и др.

Виртуализация серверов позволяет повысить доступность сервисов с помощью технологий отказоустойчивости и миграции виртуальных серверов между физическими серверами. Возможность переместить виртуальные серверы с одного физического сервера на другой без остановки, позволяет существенно повысить доступность сервиса и облегчить обслуживание всей системы. Значительно сокращается время развертывания новых сервисов - уже не требуется ждать поставки нового сервера, достаточно за несколько минут развернуть новую виртуальную машину и установить необходимое ПО. За счет того, что для виртуальных машин не требуется установка специфических драйверов, обновление прошивок и т.д. задачи администрирования также существенно упрощаются.

VMware vSphere обладает универсальной системой мониторинга состояния элементов всей системы, как на уровне физических серверов, так и на уровне виртуальных серверов предприятия. Если стандартных средств мониторинга по какимто причинам недостаточно, то существует целый ряд дополнительных приложений третьих фирм (например, Veeam Monitor), обладающих дополнительными возможностями.

Очень важно, что система позволяет распределять полномочия между администраторами, управляющими системой. Существует и технология "прозрачного" перехода от физического сервера к виртуальному, позволяющая с минимальными затратами перемещать существующий сервер в виртуальную среду, при этом пользователь не заметит каких-либо изменений и сможет продолжать работать без дополнительных модификаций (Physical to virtual).

B состав VMware vSphere (рисунок 3) входит целый ряд возможностей, позволяющих принципиально повысить надежность и управляемость виртуальной 
инфраструктуры предприятия. Поддержка этого функционала зависит от используемой редакции VMware vSphere.

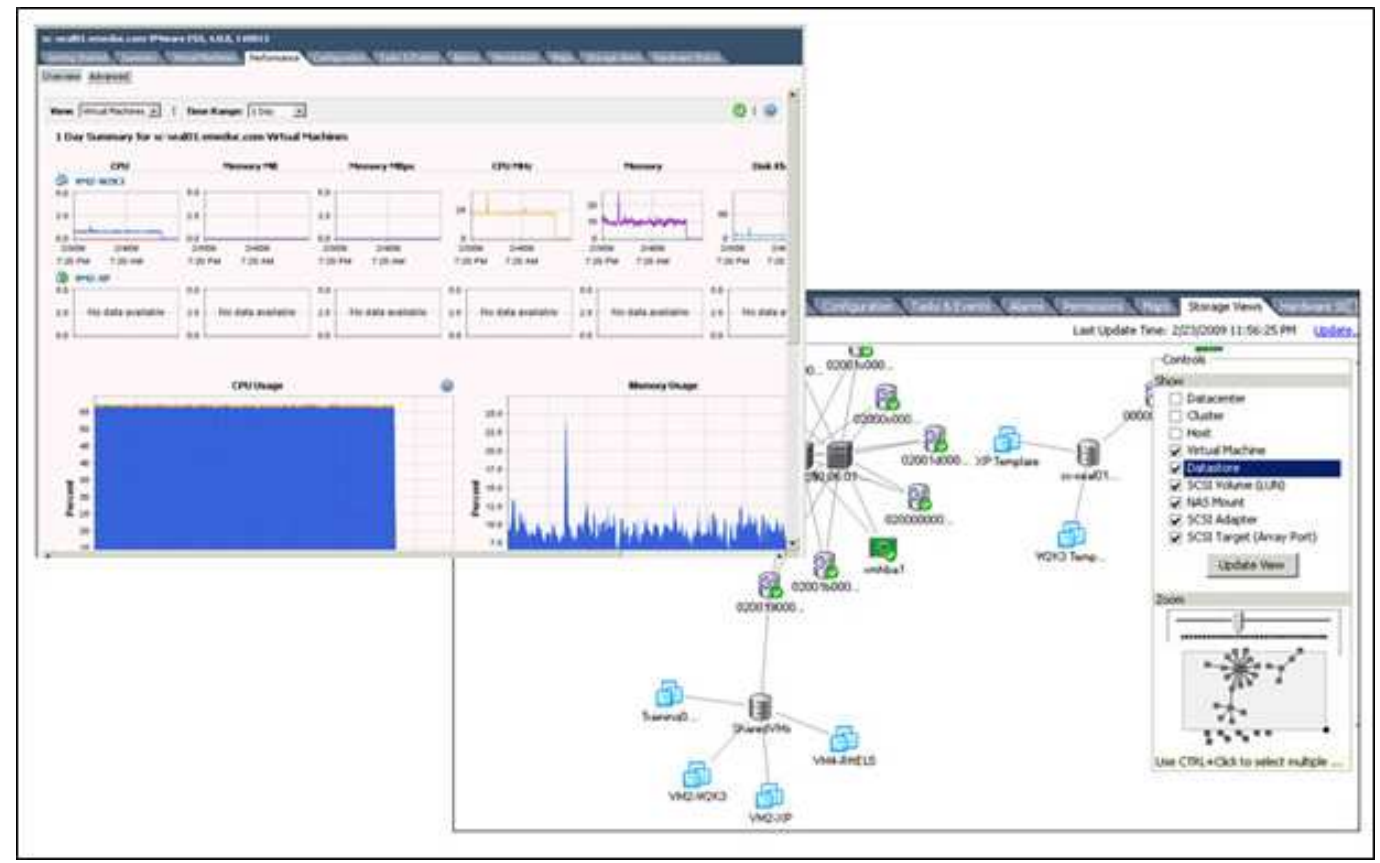

\section{Рисунок 3 - VMware vSphere}

Thin Provisioning - предоставление виртуальным серверам дискового пространства в большем объеме, чем есть на самом деле.

VC agent - управление серверами ESX/ESXi через VMware vCenter.

Update Manager - менеджер управления пакетами обновлений для серверов с установленными гипервизорами ESX/ESXi.

VMSafe - возможность установки расширенных настроек безопасности и изоляции используемых ресурсов для виртуальных машин.

vStorage APIs for Data Protection - программный интерфейс, позволяющий сторонним системам резервного копирования работать без оказания существенной нагрузки на сервер (замена системе VCB в VMware VI3). Для реализации используется технология создания мгновенных снимков виртуальных машин.

High Availability - обеспечение повышенной доступности виртуальных серверов, путем перезапуска их на резервном физическом сервере в случае отказа основного. Также есть возможность вести мониторинг конкретных сервисов, работающих внутри виртуального сервера, и производить перезапуск не только в случае сбоя оборудования, но и в случае остановки этого сервиса (рисунок 4).

Data Recovery - встроенная система резервного копирования. Позволяет управлять процессом создания и восстановления резервных копий. Целостность данных и приложений обеспечивается интеграцией с Microsoft VSS. Для гостевых систем под управлением Windows можно восстанавливать даже отдельные файлы, а не только виртуальные диски целиком. 


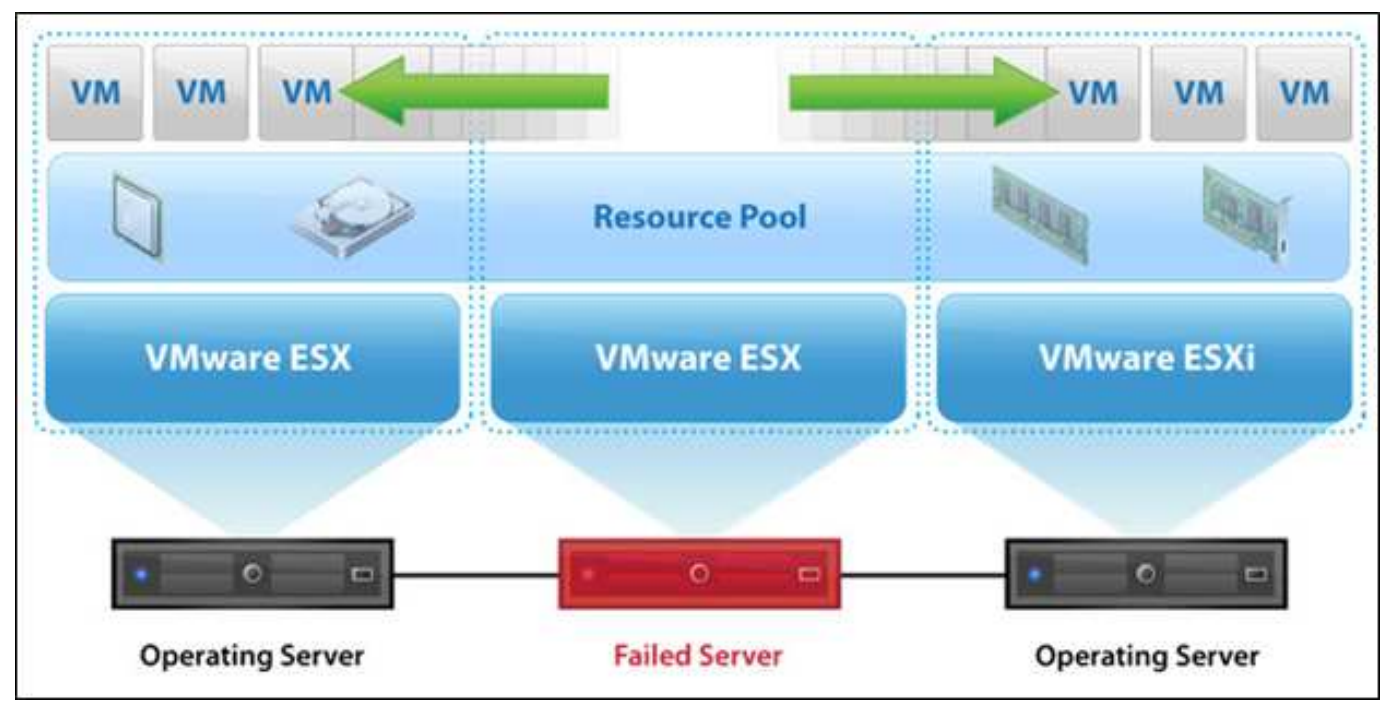

Рисунок 4 - Обеспечение непрерывности сервиса

Hot Add - поддержка добавления ресурсов (сетевых интерфейсов, памяти и т.д.) виртуальным серверам "на ходу", без остановки. Данная опция требует поддержки со стороны операционной системы на виртуальном сервере.

Fault Tolerance - обеспечение высокой доступности виртуального сервера за счет параллельного выполнения на втором физическом сервере. В случае поломки одного из них, виртуальный сервер без перерыва в обслуживании продолжит работать на втором.

vShield Zones - обеспечивает тонкую настройку безопасности виртуальных Ethernet сетей на 2/3 уровне OSI.

vMotion - позволяет мигрировать виртуальные сервера между физическими серверами без остановки работы.

Storage vMotion - позволяет перемещать диски виртуальных серверов между разными хранилищами, без остановки работы виртуальных серверов (рисунок 5).

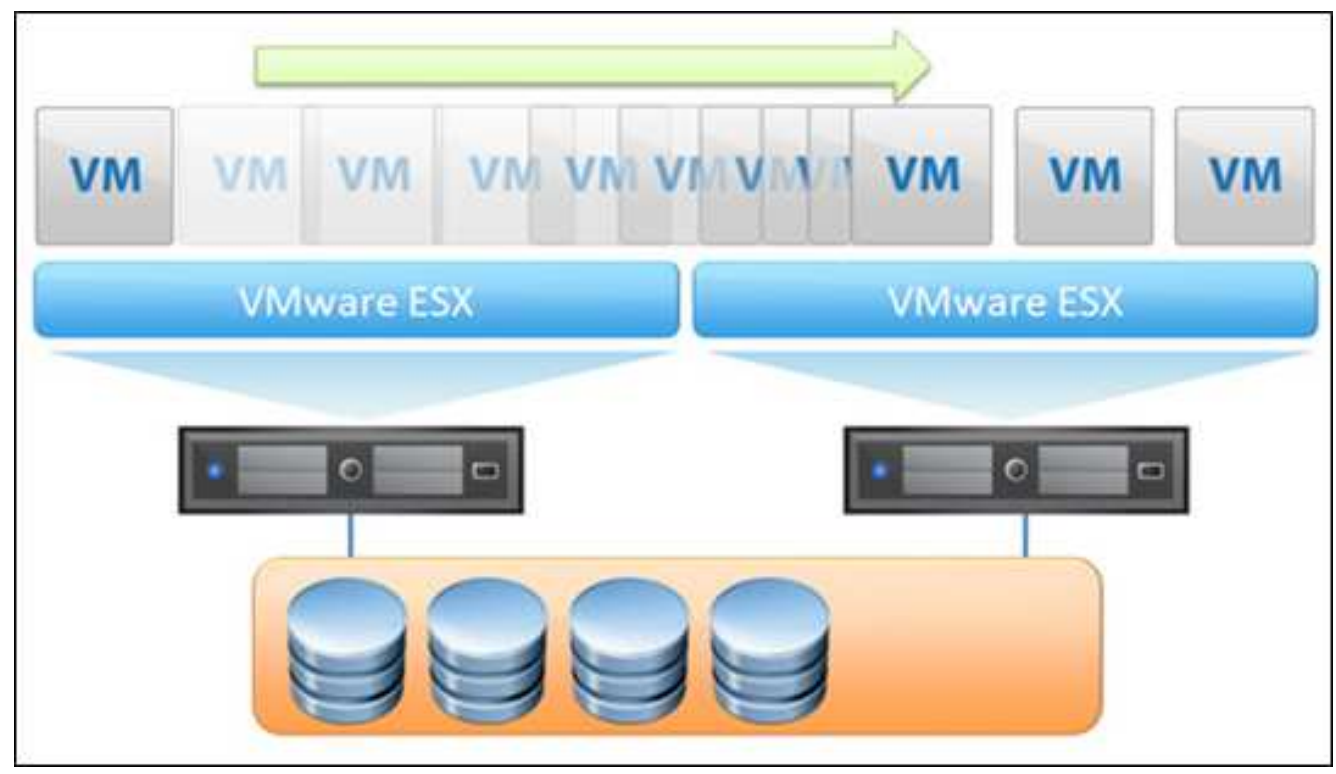

Рисунок 5 - Миграция виртуальных машин между хостами и хранилищами 
DRS+DPM - две функции, которые позволяют распределять виртуальные машины между физическими серверами в целях обеспечения максимальной эффективности использования ресурсов. При возникновении чрезмерной нагрузки на физический сервер, виртуальные машины будут перераспределены (при помощи vMotion) между серверами, имеющими свободные ресурсы. DPM позволяет выключать неиспользуемые физические сервера, а затем включать их тогда, когда в них появляется необходимость, таким образом, значительно снижается потребление электроэнергии во время низкой нагрузки на серверы.

vNetwork Distributed Switch - возможность создания виртуальных коммутаторов распределенных между разными серверами ESX. Кроме этого существует виртуальный коммутатор Cisco Nexus 1000, который является полнофункциональным программным решением от компании Cisco. Данный продукт управляется привычными для администраторов Сisco инструментами и полностью интегрируется в среду, построенную на сетевом оборудовании Cisco.

Hosted Profiles - возможность создания типовых настроек для серверов виртуализации. Позволяет централизованно управлять настройками серверов с ESX.

Third Party Multipathing - использование продуктов для балансировки и отказоустойчивости путей подключения серверов к системам хранения. Примером такого продукта является EMC Power-path.

Кроме основных программных продуктов, представляют несомненный интерес разработки компании VMware:

- VMware NSX - средство для виртуализации сетей и сетеобразующего оборудования;

- VMware vCloud Suite - программное обеспечение для организации облачных вычислений и их представления конечному пользователю как услуги;

- VMware Horizon Suite - комплекс программных средств для организации виртуальных рабочих столов изолированных рабочих компьютеров.

Применение технологии виртуализации обеспечивает еще и следующее преимущество - при определенной ограниченной мощности оборудования появляется эффект «неограниченности» предоставляемых виртуальных ресурсов с доступом в терминальном режиме. Этот эффект послужил основой нашего решения и позволил перевести процесс обучения в компьютерных классах на качественно новый уровень и остаться в пределах реального бюджета.

\section{3. Примеры практического использования}

Рассмотрим варианты практической реализации обеспечения учебного процесса с помощью технологии виртуализации.

Студенту необходимо выполнить лабораторную работу для изучения сетевого взаимодействия трех серверов различной конфигурации с различными операционными системами и различным набором программного обеспечения. 
Преподаватель создает из шаблонов виртуальные машины и передает их в управление студенту. Студент устанавливает необходимое программное обеспечение и выполняет работу в изолированной виртуальной сетевой среде.

Очевидно, что без виртуализации аппаратных ресурсов невозможно выделить каждому студенту несколько единиц дорогостоящего серверного и сетевого оборудования. Также велика вероятность того, что при выполнении практических занятий студентом будут сделаны фатальные ошибки, приводящие к краху системы и требующие значительного времени на переустановку ПО. С технологией VMware выделение ресурсов и возможность быстрого «отката» к исходному состоянию не вызывает затруднений.

Следующая задача. Группе студентов необходимо выполнить работу по инсталляции системного программного обеспечения на группе серверов, установив, допустим логический домен на базе Microsoft Windows. При обычных условиях работы, без виртуализации, выполнение этой задачи может остановить работу целой лаборатории. С применением виртуализации о том, что выполняется такая масштабная работа, будут знать только те студенты, которые ее выполняют, так как все действие происходит в изолированной виртуальной среде, где помешать кому-либо еще затруднительно.

Еще одна стандартная задача. При проведении лабораторных работ часто возникает ситуация, когда для разных групп студентов, на одних и тех же компьютерах необходимо установить программное обеспечение разных версий одного производителя, или разных производителей, но не совместимых друг с другом.

С использованием технологии виртуальных рабочих столов проблема решается созданием нужного количества виртуальных машин с набором соответствующего программного обеспечения нужных версий и производителей, а компьютер, установленный в учебной аудитории, становится только средством доступа к виртуальному рабочему столу и освобождается от целевой нагрузки. Причем местоположения такого компьютера с точки зрения доступа к виртуальным машинам безразлично.

Применение технологии виртуализации существенно упрощает процесс создания, развертывания и утилизации виртуальных машин и разделения «хостовых» ресурсов между виртуальными машинами. Создав однажды виртуальную машину, установив операционную систему и необходимый набор прикладного ПО, можно сохранить ее как шаблон. Затем, по мере необходимости, нужное количество виртуальных машин разворачивается из одного шаблона. Чтобы разграничить доступ разным группам студентов только к «своим» виртуальным машинам, можно и нужно использовать технологию ресурсных пулов (рисунок 6), когда доступ может быть предоставлен только к определенной группе виртуальных компьютеров и части дискового хранилища, которые эти виртуальные машины используют. 


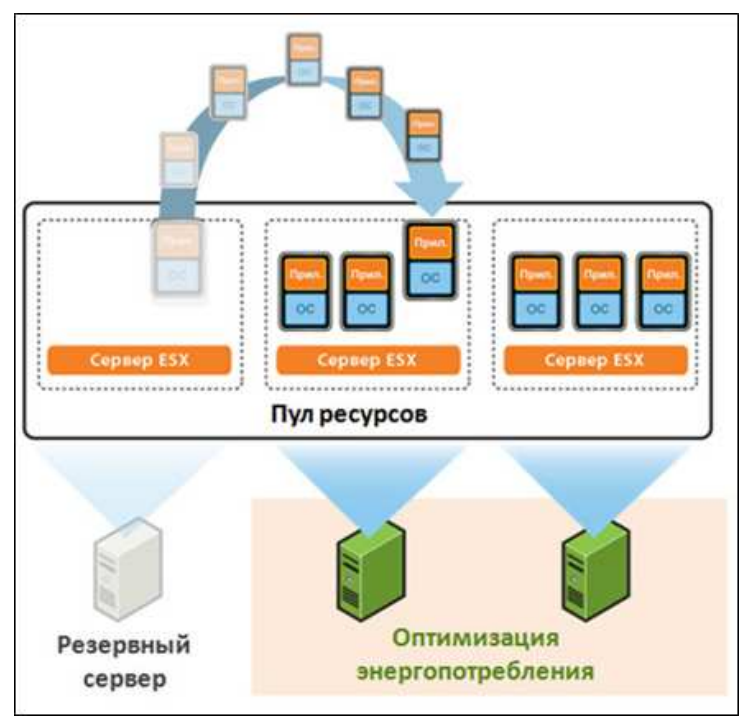

Рисунок 6 - Применение ресурсного пула

\section{4. Заключение}

В статье рассмотрена, возможность использования технологии виртуализации, для обеспечения учебного процесса на кафедре ВУЗа в условиях ограниченного финансирования. Приведены наиболее типичные задачи обеспечения учебного процесса и варианты их практической реализации.

\section{Список информационных источников}

[1] Федеральный государственный образовательный стандарт высшего профессионального образования по направлению подготовки 230100 «Информатика и вычислительная техника». [Электронный ресурс]: http://fgosvo.ru/fgosvpo/7/6/1/22.

[2] Федеральный государственный образовательный стандарт высшего профессионального образования по направлению подготовки 230400 «Информационные системы и технологии». [Электронный ресурс]: http://fgosvo.ru/fgosvpo/7/6/1/22.

[3] [Электронный ресурc]: http://www.vmware.com

[4] [Электронный ресурc]: http://www.ibm.com/ru

[5] Брагинский А.И., Виноградов В.А., Голубкова В.Б. Обоснование методики применения сервера виртуальных машин в преподавании дисциплины «КОРПОРАТИВНЫЙ И ЭЛЕКТРОННЫЙ ДОКУМЕНТООБОРОТ» // Автоматизация и управление в технических системах. - 2013. - № 3(5). С. 45-47. URL: auts.esrae.ru/5-95 (дата обращения: 22.12.2013).

[6] Николаев А.Б. Информационные технологии в менеджменте и транспортной логистике: учебное пособие / А.Б. Николаев, А.В. Остроух. - Saint-Louis, MO, USA: Publishing House Science and Innovation Center, 2013. - 254 c. - ISBN 978-0615-67110-9.

[7] Остроух А.В. Ввод и обработка цифровой информации: учебник для нач. проф. образования / А.В. Остроух. - М.: Издательский центр «Академия», 2012. - 288 с. - ISBN 978-5-7695-9457-1. 